\title{
Decreased Subcortical T2 FLAIR Signal Associated with Seizures
}

\author{
(D) P. Nicholson, (D) S. Abdulla, (D) A. Alshafai, (DD.M. Mandell, and (D)T. Krings
}

\begin{abstract}
SUMMARY: Abnormally decreased T2/T2 FLAIR signal can be seen on brain imaging of patients who are experiencing clinical or subclinical seizures and can be associated with various intracranial pathologies. We identified 29 such patients. The abnormal signal was unilateral in $75.9 \%$ of patients. It affected various lobes of the brain, but only in the anterior circulation. In 28 patients (96.6\%), there was corresponding decreased signal on DWI. The ADC was normal in all cases. In 26 patients (89.7\%), there was corresponding low signal on SWI/gradient recalled-echo; $44.8 \%$ of patients underwent contrast-enhanced scans, and there was no abnormal enhancement. Twenty-two (75.9\%) patients had documented clinical seizures on the day of imaging. The most frequent concomitant pathology was a subdural hematoma. Electroencephalograms obtained within 24 hours of imaging were available in $65.5 \%$. Findings of all of these electroencephalograms were abnormal, and these electroencephalogram changes were either localized to the area of the abnormal MR imaging signal (where the signal was unilateral) or were bilateral (where the MR imaging changes were bilateral). In summary, decreased white matter T2/T2 FLAIR signal changes can be seen in patients with remarkably similar clinical findings (particularly seizures). These changes are often correlated with abnormal electroencephalogram activity localized to the involved lobes.
\end{abstract}

ABBREVIATIONS: EEG = electroencephalogram; GRE = gradient recalled-echo

M uch has been published on the abnormal MR imaging appearances of the brain in patients experiencing acute seizures. ${ }^{1}$ These articles have mainly focused on the more common descriptions of such changes, namely diffusion restriction and $\mathrm{T} 2$ hyperintensity in the gray matter and/or subcortical white matter. ${ }^{2,3}$ Transient cortical swelling or parenchymal enhancement or both have also been described.

Less attention has been paid to decreased signal in the subcortical white matter on T2-weighted and T2-weighted FLAIR sequences. These changes have been described in various conditions such as intracranial hypotension, ${ }^{4}$ head injury, ${ }^{5}$ encephalitis, meningitis, leptomeningeal disease, ${ }^{6}$ diffuse axonal injury, and cortical ischemia ${ }^{7}$ and in patients with seizures. ${ }^{8}$ We report a series of 29 patients with abnormally decreased T2 FLAIR signal

Received August 7, 2019; accepted after revision October 2.

From the Division of Neuroradiology, Joint Department of Medical Imaging, Toronto Western Hospital, University of Toronto, Toronto, Ontario, Canada.

Please address correspondence to Patrick Nicholson, MD, Division of

Neuroradiology, Joint Department of Medical Imaging, Toronto Western Hospital, University of Toronto, 399 Bathurst St, Toronto, ON M5T 2S8, Canada; e-mail: patrick.nicholson@uhn.ca; @paddynicholson

Indicates article with supplemental on-line table.

http://dx.doi.org/10.3174/ajnr.A6328 in the subcortical white matter. We describe their imaging appearances, clinical and EEG findings, and follow-up.

\section{MATERIALS AND METHODS}

\section{Case Selection}

Using a natural-language processing search engine (mPower; Nuance, Burlington, Massachusetts), we searched our radiology data base from 2013 to 2019 for variations of the terms "T2 hypointensity" and "FLAIR hypointensity." This yielded > 350 records; these reports were then read to identify those patients with confirmed abnormal T2 FLAIR low-signal changes. Patient records for each case were then retrospectively reviewed, and demographic, clinical, and EEG features were noted. Specific clinical features recorded included the primary CNS pathology (eg, subdural hematoma) and whether the patient was having active clinically evident seizures on the day of imaging.

\section{Imaging Acquisition and Analysis}

All images were obtained at our institution on either 1.5T or 3T scanners. Each study consisted of a minimum of a sagittal T1weighted, axial T2 FSE, and axial T2-weighted FLAIR, DWI, and gradient recalled-echo (GRE)/SWI sequence (depending on the scanner). Two neuroradiologists reviewed all scans in a consensus analysis. The presence and location of abnormally decreased T2 
FLAIR signals were recorded, and the corresponding DWI and GRE/SWI signals were analyzed.

\section{RESULTS}

\section{Demographic Findings}

Patient demographics, imaging, and clinical findings for the cohort are summarized in the Table. Twenty-nine patients were

Summary of imaging and clinical findings in patients with abnormally decreased $\mathrm{T} 2$ signal

\begin{tabular}{lc}
\hline \multicolumn{1}{c}{ Findings } \\
\hline Demographics \\
Patients & 29 \\
Sex (male) & $19(65.5 \%)$ \\
Age (yr) & $49($ range, $18-85)$ \\
Clinical information & $22(75.9 \%)$ \\
Associated seizures & $14(48.3 \%)$ \\
Extrinsic compressive lesions & \\
Imaging findings & \\
Laterality & $7(24.1 \%)$ \\
Bilateral & $22(75.9 \%)$ \\
Unilateral & $21(72.4 \%)$ \\
Multiple lobes involved & $19(65.5 \%)$ \\
Frequency of involved lobes & $17(58.6 \%)$ \\
Frontal & $16(55.2 \%)$ \\
Parietal & $14(48.3 \%)$ \\
Temporal & \\
Occipital & $19(65.5 \%)$ \\
EEG findings & \\
Availability & $12 / 12$ Cases with MRI T2 \\
Laterality & changes unilateral \\
Unilateral & $7 / 7$ Cases with MRI T2 \\
Bilateral & changes bilateral \\
Follow-up imaging & $9(31 \%)$ \\
Availability & $6(66.6 \%)$ \\
Changes resolved & $4 / 6$ \\
Associated with & \\
compressive lesion & $3(33.3 \%)$ \\
Nonresolved/gliosis & $1 / 3$ \\
Associated with & \\
compressive lesion & \\
\hline & \\
\hline & \\
\hline & \\
\hline & \\
\hline
\end{tabular}
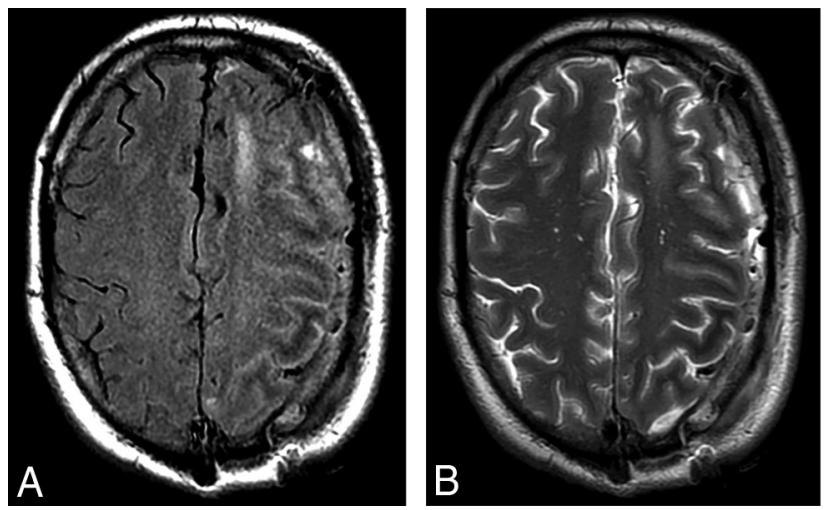

identified; 19 were men (65.5\%). The median patient age was 49 years (range, $18-85$ years).

\section{Imaging Findings}

Imaging findings are summarized in the Table. Hypointensity on T2 and T2 FLAIR images was present in the subcortical white matter in all cases, with sparing of the overlying cortex (Figs 1-3). The abnormal signal was bilateral in 7 cases $(24.1 \%)$ and unilateral in 22 (75.9\%). It was usually found in $>1$ lobe. In 6 cases $(20.7 \%)$, it was confined to the frontal lobe only; in 1 case $(2.1 \%)$, to just the parietal lobe; and in 1 case, to the occipital lobe alone. Overall, the frontal lobe was the most common lobe involved (19 cases, $65.5 \%$ ), followed by the parietal $(17 \%, 58.6 \%)$, temporal (16\%, 55.2\%), and occipital $(14 \%, 48.3 \%)$ lobes. The signal pattern was not seen in the posterior fossa in any case. In 28 patients (96.6\%), the areas of brain with low T2 signal showed corresponding decreased signal on DWI and increased DWI signal in none. The ADC findings were normal in all cases. In 26 patients (89.7\%), there was also corresponding decreased signal on SWI/GRE sequences. Contrast was administered in 13 scans (44.8\%); none of the T2 hypointense signal areas showed enhancement. There were no instances of T2 FLAIR hyperintense cortical signal.

\section{Clinical Data}

Twenty-seven patients were inpatients at the time of imaging (93.1\%), while the remaining 2 were imaged as outpatients (6.9\%). All clinical and imaging data are presented in the On-line Table. All the inpatient MR imaging studies were performed within 72 hours of the initial clinical presentation. Twenty-two patients $(75.9 \%)$ had documented clinical seizures on the day of imaging. Of those patients with seizures, 18 seizures were generalized $(81.8 \%)$, while the remaining $4(18.2 \%)$ were focal. A further 3 patients had a documented alteration in the level of consciousness and were undergoing investigation for "possible seizures." Of the remaining patients, 1 patient had asymptomatic signal changes adjacent to a meningioma, 2 patients developed a subdural hematoma following a meningioma resection but were also asymptomatic, and the remaining 2 patients had these signal changes underlying an acute subdural hematoma and were, similarly, clinically asymptomatic. The most frequent concomitant
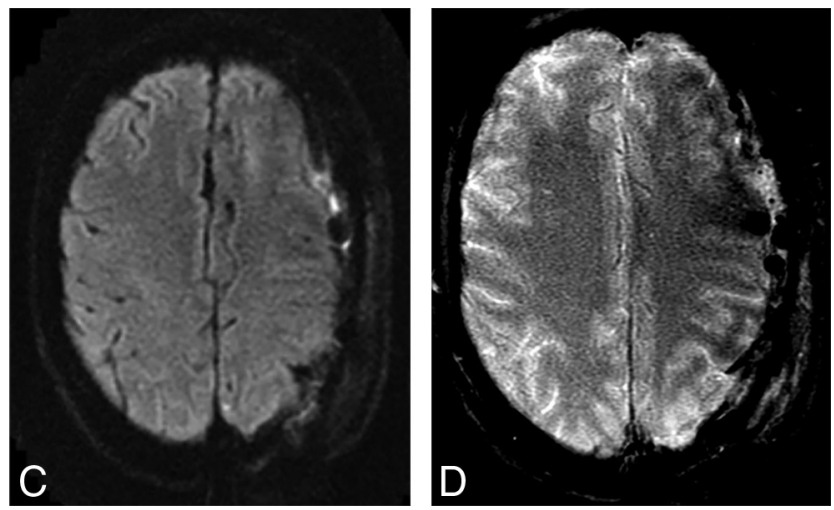

FIG 1. Patient admitted to the intensive care unit with persistent seizures 1 day after evacuation of a left-sided subdural hematoma. $A$, T2 FLAIR, $B$, FSE T2, C, DWI, and D, GRE-weighted images. Note the decreased T2-weighted signal in the subcortical white matter of the left frontal and parietal lobes on $A$ and $B$, as well as the lack of corresponding diffusion restriction on $C$. $D$, GRE-weighted image shows there is subtle corresponding decreased subcortical white matter signal change. 

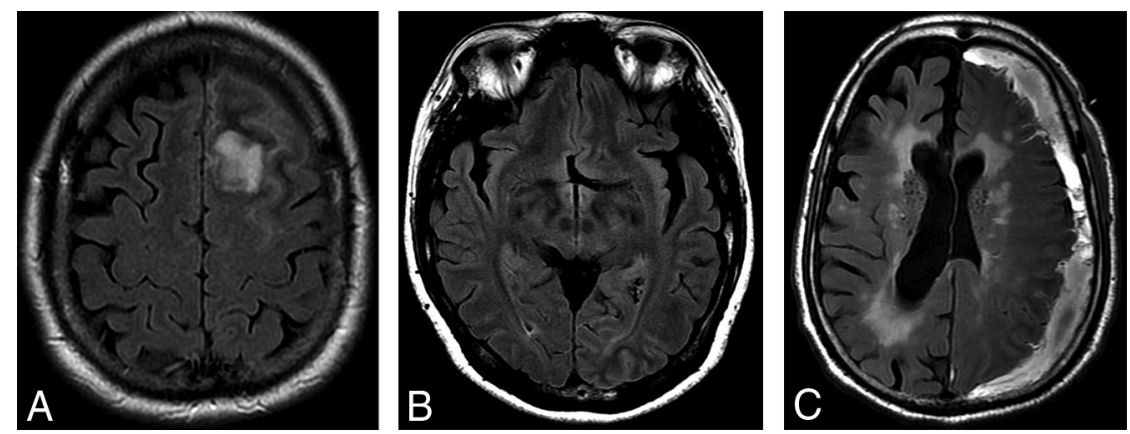

FIG 2. A, T2 FLAIR images from a 49-year-old male patient being followed for a left-frontal meningioma with asymptomatic low signal in the subcortical white matter adjacent to the meningioma. Nonspecific failure of suppression of CSF signal in the subjacent sulci was also noted. B, T2 FLAIR images showing low signal in the left occipital lobe in a 46-year-old patient who initially presented with visual seizures. C, T2 FLAIR images from a 48-year-old patient with a left convexity subdural hematoma and seizures. Subcortical low $\mathrm{T} 2$ signal is present in the compressed posterior frontal and parietal lobes.
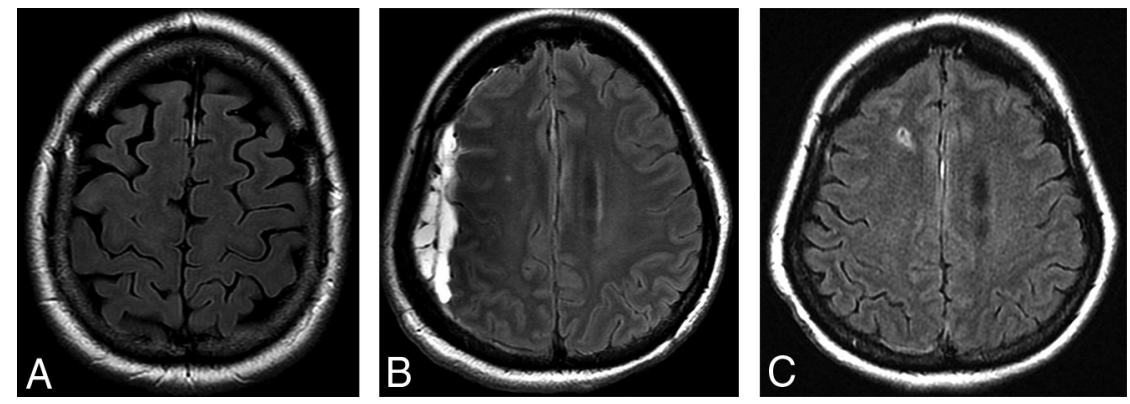

FIG 3. $A, T 2$ FLAIR image in the same patient as in Fig $2 A$ shows complete resolution of the white matter changes 2 years after resection of the lesion. B, T2 FLAIR image from a 53-year-old female patient with a spontaneous right-sided acute subdural hematoma. T2 hypointense signal changes are present in the subjacent white matter. $C$, Follow-up 5 months later shows resolution of both the hematoma and the white matter changes.

pathology was a subdural hematoma (12 patients, $41.8 \%$ ), and the signal alterations were always found in the subjacent white matter. Similarly, the signal abnormality was always found in the white matter adjacent to compressed brain region in those cases of meningioma. Overall, an extrinsic compressive lesion (eg, meningioma, subdural hematoma) was present in 14/29 patients (48.3\%).

Electroencephalograms (EEGs) obtained within 24 hours of the MR imaging were available in 19 of the total cohort (65.5\%), including all patients who were having clinical seizures. In these patients, abnormal EEG signal was found in all cases, and these changes were localized to the side of the MR imaging abnormality in 12/12 cases in which the MR imaging signal was unilateral. The EEG showed bilateral abnormalities in the remaining 7 patients who had bilateral T2-hypointense changes. All inpatients were receiving antiseizure medication at the time of MR imaging. Two outpatients were taking antiseizure medication, while data were unavailable for the remainder.

\section{Follow-Up}

Imaging follow-up was available in 9 patients (31\%), at a median of 18 months (range, 1.25-72 months). The changes had resolved in
6 of these patients $(66.6 \%$ of this subgroup), while 2 patients had persistent decreased T2 FLAIR signal (at 18- and 5month follow-ups, respectively). One patient had gliosis with volume loss on follow-up imaging (performed at 18 months). Of those patients in whom the changes resolved, 4/6 (66.6\%) had an extra-axial compressive lesion, which had been removed or treated in the interval. Conversely, 1 of the 3 patients in whom the signal changes did not resolve or progressed to gliosis had a compressive lesion which was treated.

\section{DISCUSSION}

We have described a series of patients with remarkably similar imaging features: All had areas of decreased T2 FLAIR signal in the subcortical white matter, usually with corresponding decreased signal on GRE/SWI and without corresponding diffusion restriction or enhancement. This imaging pattern was most commonly unilateral and affected multiple lobes. In addition, many of these patients had strikingly similar clinical features: More than $80 \%$ had either clinical seizures or altered levels of consciousness at the time of imaging. We do not perform continuous EEG monitoring on all patients in our intensive care unit, so it is also possible that at least some of the remaining patients were experiencing some subclinical seizures during the course of their admission. The causative factor was also quite similar in many cases; almost half (48\%) had an extrinsic lesion (subdural hematoma, meningioma) with mass effect on the subjacent subcortical white matter.

The question still exists regarding the nature of these white matter signal alterations. It is not clear whether they are the result of the causative process that leads to the seizure activity or whether they represent imaging evidence of abnormal neuronal activity "spilling over" to the adjacent white matter-ie, a possible imaging biomarker of seizure activity. As mentioned in our introduction, there are a range of pathologies that can cause decreased T2 signal in white matter. For example, this pattern can be seen in patients with a primarily leptomeningeal process, such as meningitis or leptomeningeal metastasis. However, it can also be seen in patients with intracranial hypotension, which is not a primary "leptomeningeal" pathology per se but still could be considered an extra-axial issue that is having an effect on intra-axial tissues. In addition, these disparate diseases often cause generalized signal changes. In our cohort, we see a remarkable side concordance (and often lobe concordance) with both causative pathology (when such a cause was known) and with abnormal EEG activity. 
Previously, some groups have attributed this signal change to accumulation of oxygen-free radicals in the white matter, ${ }^{9}$ but the evidence for this theory remains sparse at best. Free radicals are transient oxygen intermediates, with a supposed paramagnetic effect due to their unpaired electrons. However, recent laboratory work has shown that the paramagnetic effect of reactive oxygenfree radicals is mainly on T1 shortening, with a negligible effect on T2-relaxation times. ${ }^{10}$ If these transient free radicals are responsible for this decreased signal, we would not expect to see persistent changes many months later as we did in some patients in our cohort. Furthermore, in patients with seizures, free radicals are present in the cortex as well as in the subcortical white matter, ${ }^{11}$ yet we do not see these signal changes in the cortex in our patients. On balance, it appears that there is not much proof to directly implicate free radicals as the cause of the described findings.

Some other possibilities remain. It is interesting that there was associated decreased signal on $\mathrm{T}^{\star}$-weighted sequences in our cohort. Decreased signal on such sequences has been associated with increased oxygen extraction (ie, an uncoupling between oxygen supply and demand). Cortical perfusion abnormalities are well-described in patients undergoing seizure activity ${ }^{12}$ as well as with pathologies such as subdural hematomas, ${ }^{13}$ so perhaps the changes we are seeing are reflective of such increased oxygen extraction. Direct nonheme iron deposition in the tissues has also been suggested previously, ${ }^{7,9}$ and while this could account for the pattern we are seeing acutely, it would not be expected to resolve in most cases as it did in our cohort.

The neurochemical changes in the subcortical white matter in a patient group such as ours are undoubtedly complex. Various pathophysiologic mechanisms are likely at play, such as loss of autoregulation, changes in cellular permeability, regional perfusion changes, and neuronal excitotoxicity. ${ }^{14}$ Much has been written about these changes ${ }^{15,16}$ and about the danger of applying a single imaging label to what is surely multiple parallel processes. ${ }^{17}$ However, it appears clear that the MR imaging findings we describe indicate some abnormality in the subcortical white matter. They may also serve as a useful suggestion to the clinician to consider EEG monitoring in those patients in whom seizures cannot be clinically identified (eg, the intubated patients in the intensive care unit).

There are obvious limitations with this retrospective study, which was performed in an adult-only population. It is possible that the abnormal EEG activity could be related to the offending pathology in such cases as subdural hematomas; however, this EEG-imaging concordance was also maintained in those patients in whom such an extrinsic factor did not exist (eg, those patients with primary seizures or epilepsy). We did not perform advanced imaging techniques such as MR perfusion, which could potentially provide more information about the areas of signal alteration. It is also likely that these changes are also underreported, both in our center and in the literature more generally, so the prevalence of such changes is possibly higher than that seen here. Finally, most of our patients were inpatients (93.1\%), so we are unable to comment on the prevalence of these findings in outpatients with chronic seizure disorders.

\section{CONCLUSIONS}

We have described a cohort of 29 patients with a similar clinical presentation (most had seizures/altered level of consciousness), with strikingly similar imaging findings of decreased T2-weighted signal in the subcortical white matter, in whom EEG abnormalities were frequently localized to the area of the MR imaging changes. We emphasize that these changes are often correlated with a degree of seizure activity in the surrounding brain parenchyma. Whether the altered signal change is a cause or effect of seizure activity is still uncertain, as is the underlying pathophysiology causing the signal changes.

Disclosures: Timo Krings—UNRELATED: Consultancy: Stryker, Medtronic, Penumbra; Royalties: Thieme; Stock/Stock Options: Marblehead.

\section{REFERENCES}

1. Cianfoni A, Caulo M, Cerase A, et al. Seizure-induced brain lesions: a wide spectrum of variably reversible MRI abnormalities. Eur J Radiol 2013;82:1964-72 CrossRef Medline

2. Cartagena AM, Young GB, Lee DH, et al. Reversible and irreversible cranial MRI findings associated with status epilepticus. Epilepsy Behav 2014;33:24-30 CrossRef Medline

3. Giovannini G, Kuchukhidze G, McCoy MR, et al. Neuroimaging alterations related to status epilepticus in an adult population: definition of MRI findings and clinical-EEG correlation. Epilepsia 2018;59(Suppl 2):120-27 CrossRef Medline

4. Adachi M, Mugikura S, Shibata A, et al. Relative decrease in signal intensity of subcortical white matter in spontaneous intracranial hypotension on fluid-attenuated inversion recovery images. AJNR Am J Neuroradiol 2009;30:906-10 CrossRef Medline

5. Okanishi T, Saito Y, Fujii S, et al. Low signal intensity and increased anisotropy on magnetic resonance imaging in the white matter lesion after head trauma: unrecognized findings of diffuse axonal injury. J Neurol Sci 2007;263:218-22 CrossRef Medline

6. Lee JH, Na DG, Choi KH, et al. Subcortical low intensity on MR images of meningitis, viral encephalitis, and leptomeningeal metastasis. AJNR Am J Neuroradiol 2002;23:535-42 Medline

7. Ida M, Mizunuma K, Hata $\mathrm{Y}$, et al. Subcortical low intensity in early cortical ischemia. AJNR Am J Neuroradiol 1994;15:1387-93 Medline

8. Raghavendra S, Ashalatha R, Thomas SV, et al. Focal neuronal loss, reversible subcortical focal $\mathrm{T} 2$ hypointensity in seizures with a nonketotic hyperglycemic hyperosmolar state. Neuroradiology 2007;49:299-305 CrossRef Medline

9. Cross PA, Atlas S, Grossman R. MR evaluation of brain iron in children with cerebral infarction. AJNR Am J Neuroradiol 1990;11:34148 Medline

10. Tain RW, Scotti AM, $\mathrm{Li}$ W, et al. Imaging short-lived reactive oxygen species (ROS) with endogenous contrast MRI. J Magn Reson Imaging 2018;47:222-29 CrossRef Medline

11. Shin EJ, Jeong JH, Chung YH, et al. Role of oxidative stress in epileptic seizures. Neurochem Int 2011;59:122-37 CrossRef Medline

12. Newton MR, Berkovic SF, Austin MC, et al. Postictal switch in blood flow distribution and temporal lobe seizures. J Neurol Neurosurg Psychiatry 1992;55:891-94 CrossRef Medline

13. Brodersen P, Gjerris F. Regional cerebral blood flow in patients with chronic subdural hematomas. Acta Neurol Scand 1975;51:23339 CrossRef Medline

14. Holmes GL. Seizure-induced neuronal injury: animal data. Neurology 2002;59:S3-6 CrossRef Medline

15. Cole AJ. Status epilepticus and periictal imaging. Epilepsia 2004;45 (Suppl 4):72-77 CrossRef Medline

16. Briellmann RS, Wellard RM, Jackson GD. Seizure-associated abnormalities in epilepsy: evidence from MR imaging. Epilepsia 2005;46:760-66 CrossRef Medline

17. Grillo E. Seizure-induced excitotoxicity: just considering it could be a fallacy-comment on: Cianfoni A, Caulo M, Cerase A. Seizure-induced brain lesions: a wide spectrum of variably reversible MRI abnormalities (in press). Eur J Radiology 2013;82:e903-04 CrossRef Medline 